\title{
Editorial
}

\section{And Yet Another New Year's Resolution}

\section{Pushing EJPA's Advance Articles}

\author{
Samuel Greiff
}

Institute of Cognitive Science and Assessment (COSA), University of Luxembourg, Luxembourg

A new year, 2019, has just started and everywhere you turn, you will find flashbacks of 2018, plans and thoughts on how 2019 will be different from 2018, and, of course, New Year's resolutions! And - spoiler alert - this editorial won't hold back on New Year's resolutions either.

What virtually all New Year's resolutions have in common is that we want to do something better than in the past: Do more sports, eat healthier, spend more time with the family, write more manuscripts, procrastinate less, and so forth. Despite the good nature of New Year's resolutions, attempts towards increasing their chance of success seem to work moderately at best (Alan Marlatt \& Kaplan, 1972; Oscarsson, Rozental, Andersson, \& Carlbring, 2017). However, their popularity remains unbroken and even journals, or at least their editors, tend to have New Year's resolutions (e.g., Woodruff, 2019). EJPA is no different from this, but our New Year's resolutions are specific enough to have a high likelihood of becoming reality - and they have implications for readers and authors.

The core New Year's resolution for EJPA is to reduce the number of published advance articles, often called "onlinefirst articles", that we have accumulated over the years, and to assign them more quickly to an issue. Not a new idea, by the way. In fact, the increase in the number of issues per year from 4 to 6 in 2017 was targeted at reducing the number of articles that had not been assigned to an issue yet, but due to the high number of submissions and their high quality, this attempt utterly failed. Thus, as we move into 2019 we are still faced with a substantial number of advance articles. This means that after acceptance of an article, it will take over 24 months until the paper is assigned to an issue and receives page numbers even though it is finalized, available online (including a doi), and citable usually no later than 6 months after acceptance. In a way, this is a bit like having a new novel (personally, I have been waiting for the sequels of some novels for plenty of years...) that is available as e-book, but then it takes several years until the regular paper version is out. And sometimes it is just nicer to feel the paper in your hands... but there are other reasons why it is important to authors that their manuscript is assigned to an issue.

What does this mean specifically? First of all, obviously we will continue the six issues per year cycle, but in addition to this, as a temporary measure, Hogrefe, the publishing house of EJPA, has agreed to publish issues of double their usual length in 2019, so this year - thanks to the publisher - you will find thicker issues in your mailbox. This will help EJPA to reduce the time between acceptance of an article and its assignment to a specific issue to a matter of a few weeks or months, in line with what you see in many other major journals as well. For authors, this means that their papers are assigned much quicker to an issue and, for readers, it means that you might want to allocate more time to assessment-related topics and to the papers you will find in EJPA in 2019.

Related to this, my personal New Year's resolution is among many other things such as leading a healthy life and being a better person - to not write lengthy editorials and at least for this one, this worked out. So, we have two resolutions in this editorial, both specific and actionable: (1) decreasing backlog and (2) not writing extensive editorials. Interestingly, one is mastery-oriented, the other avoidance-oriented, in line with a study that shows that around $2 / 3$ rds of all New Year's resolutions are masteryoriented and 1/3 avoidance-oriented (Woodruff, 2019).

But wait, what about your New Year's resolution? In case you haven't made any and you need some suggestions, how about these:

- Continue to submit your best work to EJPA. We are looking forward to receiving your manuscripts;

- Attend the 15th ECPA in Brussels, Belgium July 7-10, 2019 and meet great colleagues; 
- Get involved, be it in EAPA as the association housing the journal or in EJPA as its flagship journal, be it as author, reviewer, board member, reader. . ;

- Give us feedback: What would you like to see improved in the journal? Where are we doing a good job? Let us know at ejpaeditor@gmail.com.

Whichever of the above you choose, the editorial team of EJPA sends best wishes for a prosperous year 2019.

\section{References}

Alan Marlatt, G., \& Kaplan, B. E. (1972). Self-initiated attempts to change behavior. A study of New Year's resolutions. Psychological Reports, 30, 123-131. https://doi.org/10.2466/pr0.1972. 30.1 .123
Oscarsson, M., Rozental, A., Andersson, G., \& Carlbring, P. (2017). New Year's resolutions. A large-scale randomized controlled trial. Paper presented at the 9th Swedish Congress on Internet Interventions, Linköping, Sweden, November 3, 2017.

Woodruff, T. K. (2019). New Year's resolutions; New year reviewers; New year of review. Endocrinology, 60, 36-37. https://doi. org/10.1210/en.2018-01003

\section{Samuel Greiff}

Institute of Cognitive Science and Assessment (COSA)

University of Luxembourg

11, Porte des Sciences

4366 Esch sur Alzette

Luxembourg

samuel.greiff@uni.lu 